\title{
NEUTRON BURSTS IN \\ A RING REACTOR
}

October 1958

CONTRACT AT-11-1-GEN-14

BETTIS PLANT

PIITSBURGH， PENNSYIVANIA

Operated for the

U.S. ATOMIC ENERGY COMMISSION bY BETTIS ATOMIC POWER DIVISION, WESTINGHOUSE ELECTRIC CORPORATION 


\section{DISCLAIMER}

This report was prepared as an account of work sponsored by an agency of the United States Government. Neither the United States Government nor any agency Thereof, nor any of their employees, makes any warranty, express or implied, or assumes any legal liability or responsibility for the accuracy, completeness, or usefulness of any information, apparatus, product, or process disclosed, or represents that its use would not infringe privately owned rights. Reference herein to any specific commercial product, process, or service by trade name, trademark, manufacturer, or otherwise does not necessarily constitute or imply its endorsement, recommendation, or favoring by the United States Government or any agency thereof. The views and opinions of authors expressed herein do not necessarily state or reflect those of the United States Government or any agency thereof. 


\section{DISCLAIMER}

Portions of this document may be illegible in electronic image products. Images are produced from the best available original document. 


\section{LEGAL NOTICE}

This report was prepared as an account of Government sponsored work. Neither the United States Navy, nor the Commission, nor any person acting on behalf of these agencies:

A. Makes any warranty or representation, express or implied, with respect to the accuracy, completeness, or usefuiness of the information contained in this report, or that the use of any information, apparatus, method, or process disclosed in this report may not infringe privately owned rights; or

B. Assumes any liabilities with respect to the use of, or for damages resulting from the use of any information, apparatus, method, or process disclosed in this report.

As used in the above, "person acting on behalf of the Commission or the U. S. Navy" includes any cmployee or contractor of these agencies to the extent that such employee or contractor prepares, handles or distributes, or provides access to, any information pursuant to his employment or contract with the Commission or the U, S. Navy. 
WAPD-TM-164

(UC-34, Physics and Mathematics TID-4500, 14th. Edition)

NEUTRON BURSTS IN A RING REACTOR

by

D. R. Harris

October 1958

Contract AT-11-1-GEN-14

\section{Printed in U. S. A. Price .50

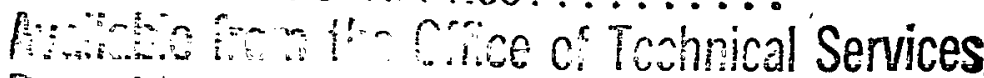

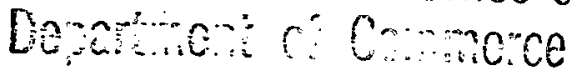 \\ Vashingion 25, D.C. \\ NOTE}

This document is an interim memorandum prepared primarily for internal reference and does not represent a final expression of the opinion of Westinghouse. When this memorandum is distributed externally, it is wi th the express 'understanding that. Westinghouse makes no representation as to completeness, accuracy, or usability of information contained therein。

Operated for the U. S. A Luilic Eriergy Commission by 
SPECIAL EXTERNAL DISTRIBUTION

Manager, Pittsburgh Naval Reactors Operations Office,

$$
U_{0 .} \text { S. A tomic Energy Commission }
$$

John Orndbff, Los Alamos Scientific Laboratory

INTERNAL DISTRIBUTION.

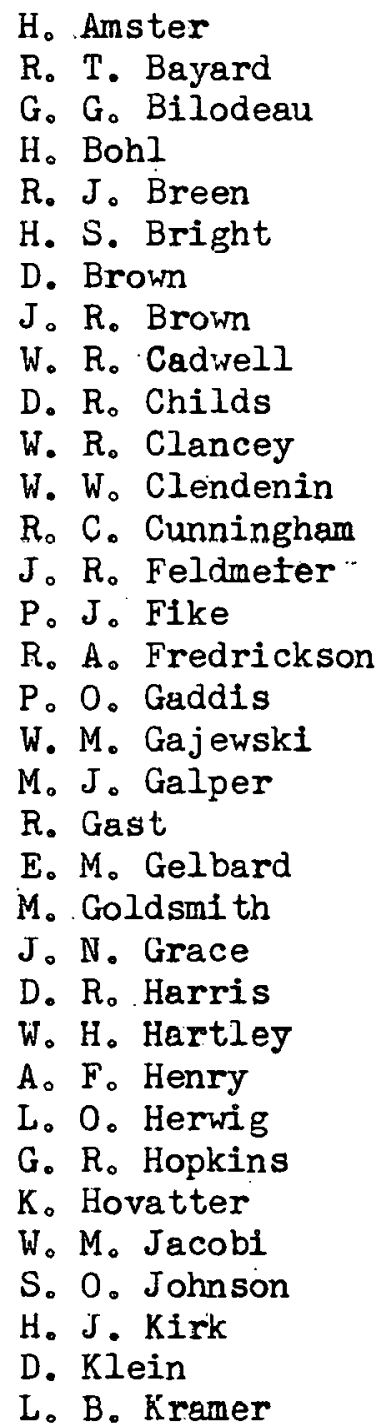

$J$. H. Leonard

S. H. Levine

O. J. Marlowe

C. W. Maynard

J. E. Meyer

B. H. Mount

E. J. Ney (NRF)

B. H. Noordhof $f$

H. F. Raab

K. E. Relf

P. F. Rose

S. Sandhaus (NRF)

E. R. Sanford

R。 R. Schiff

F. Schwoerer

J. W. Simpson

$J$. Spanier

S. Stein

M. R. Stuart

J. J. Taylor

W. R. Thompson

R. R. Thomson

R. S. Varga

W. F。 Vogelsang

$T$ o $J$ 。 Walker

R。 S。Wick

W. F。Witzig

J。L。Wolff

O. J . Woodruff

J. M. Yadon (NRF)

Document Library

NRF Library 


\section{TABLE OF CONTENTS}

$\underline{\text { Page }}$ ABSTRACT ............................. .

I. INTRODUCTION ........................ I

II... GENERAL SOLUTION ....................... 2

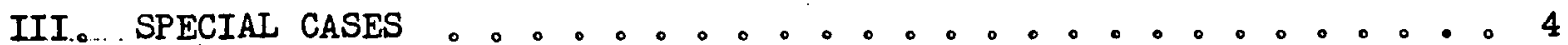

A. Delayed Neutrons Absent。 ............... 4

B. Critical Reactor With One Delayed Neutron Group. . . . . . 6

IV. DISCUSSION 。. . . . . . . . . . . . . . . . . 7

REFERENCES 。. . . . . . . . . . . . . . . . . 9 


\section{LIST OF FIGURES}

Page

Figure 1. Shape Function of Prompt Neutron Distribution Resulting From Neutron Burst 。. . . . . . . ......... 10

Figure 2. Neutron Density at Point of Neutron Burst and at Opposite Side of Ring Reactor Versus Time After Burst 。 . . . . . 11 


\section{ABSTRACT}

Azimuthal propagation of disturbances in a large annular reactor is investigated by studying the equalization of neutron bursts in a ring or simply-periodic reactor. One-velocity diffusion theory is employed to obtain convenient expressions for the time required for approximate equalization of neutron density around the ring. 


\section{INTRODUCTION}

When a burst of neutrons is injected into a uniform reactive medium the resulting neutron distribution usually is not separable as a product of a function of time and a function of the spatial coordinates. As a result, computation of the non-separable distribution is difficult unless the distribution can be expressed as a tabulated transcendental function. For example, the neutron density resulting from injection of a burst of neutrons into an infinite or semi-infinite medium sometimes can be expressed in terms of tabulated Gaussian functions. In particular this is the case when one-velocity neutrons diffuse through the medium, and in the same approximation this study deals with another geometry in which the non-separable solutions are tabulated functions. The geometry is that of the ring or simply-periodic system, where if I is the circumference of the ring and $n(x, t)$ is the neutron density at time $t$ and at location $x$ measured around the ring, then $n(x, t)=n(x+L, t)$. Such a system can be regarded as a first approximation in the investigation of azimuthal propagation of disturbances in a large annular reactor.

In Section II is presented the solution for the mean neutron density following injection of a neutron or delayed neutron precursor at some point on the ring. As is the usual case when delayed neutrons are included in the model, the solution is not easily computed. When, however, in Section III the delayed neutrons are neglected it is found that the solution is expressed in terms of a tabulated $\theta$-function. A numerical example is presented, and a simple expression is found for the .time required for approximate equalization of the prompt neutron densi.ty around the ring. Finally in section III the case of a single type of delayed neutrons is discussed. When this single delayed group is given the average characteristics of all delayed neutrons emitted from fission, it is found 
that the time required for equalization of neutron density around a critical reactor is of the order of magnitude of the precursor decay time of course when equalization has taken place the problem becomes separable, $i_{0} e_{0}$, the ring. reactor's uniform neutron content is a function of time alone. The results are summarized in Section IV.

\section{GENERAL SOLUTION}

When one-velocity neutrons diffuse around the ring the mean neutron density $n(x, t)$ satisfies the following equations:

$$
\begin{aligned}
& {\left[-\frac{\partial}{\partial t}+M^{2} \tau_{a}^{-1} \frac{\partial^{2}}{\partial x^{2}}+\left(k_{0}-1\right) \tau_{a}^{-1}\right] n(x, t)+\sum_{i=1}^{I} \lambda_{1} c_{i}(x, t)=0} \\
& {\left[-\frac{\partial}{\partial t}-\lambda_{i}\right] c_{i}(\dot{x}, t)+k_{i} \cdot{ }_{a}^{-1} n(x, t)=0 ; i=1,2, \ldots, I}
\end{aligned}
$$

Here $M$ is the migration length, $\tau_{a}$ is the mean time for loss of a neutron by absorption. or by radial and axial leakage, $c_{i}(x, t)$ is the density of 1 -th type delayed neutron precursors, and $\lambda_{i}$ is the decay constant of the 1-th type precursar... If $\bar{v}_{i}$ represents the average number of prompt $(i=0)$ or latent ( $1 \neq 0$ ) nentrons formed in a fission, and if $\tau_{f}$ is the mean time required for production of a fisision by a neutron, then $k_{1}$ is defined as $\bar{v}_{1} \tau_{f}^{-1} \tau_{a}$. No neutrons can be lost to the system by leakage along the $x$-axis; hence $k_{1}$ represents the effective prompt $(1=0)$ or delayed $(1 \neq 0)$ multiplication factor.

If a neutron is injected into the ring at $x=0, t=0$, the resulting average neutron and precursor densities must be expressible as pure cosine series:

$$
n(x, t)=\sum_{j=0}^{\infty} N_{j}(t) \cos j 2 \pi \frac{x}{L} ; c_{i}(x, t)=\sum_{j=0}^{\infty} c_{1 j}(t) \cos j 2 \pi \frac{x}{L}
$$


Substituting Eqs: (2) into Eqs. (1) it is found that the coefficients $N_{j}(t)$ and $C_{i j}(t)$ satisfy:

$$
\begin{aligned}
& -\stackrel{N}{N}_{j}(t)+\left(k_{0}-1-j^{2} 4 \pi^{2} M^{2} / L^{2}\right) \tau_{a j}^{-1} N_{j}(t)+\sum_{1=1}^{I} \lambda_{1} C_{i j}(t)=0 \\
& -\dot{C}_{1 j}(t)-\lambda_{1} C_{1 j}(t)+k_{1} \tau_{a}^{-1} N_{j}(t)=0
\end{aligned}
$$

Solving Eqs. (3) by the usual method of Laplace transformit we find:

$$
N_{j}(t)=\sum_{q=1}^{I+1} e^{a} \frac{d q}{t}\left[A_{0 j q} N_{j}(0)+\sum_{i=1}^{I} A_{i j q} C_{i j}(0)\right]
$$

where the real numbers $a_{j q}$ are roots of the algebraic equations:

$$
a_{j}-\left(k_{0}-1-j^{2} 4 \pi^{2} M^{2} / L^{2}\right) \tau_{a}^{-1}-\sum_{i=1}^{I} k_{i} \tau_{2}^{-1} \lambda_{1} /\left(a_{j}+\lambda_{i}\right)=0
$$

and by definition:

$$
\begin{aligned}
& A_{0 j q} \equiv\left[1+\sum_{m=1}^{I} k_{m} \tau_{a}^{-1} \lambda_{m} /\left(\alpha_{j q}+\lambda_{m}\right)^{2}\right]^{-1} ; A_{i j q} \equiv A_{0 j q} \lambda_{1} /\left(\alpha_{j q}+\lambda_{f}\right) \\
& \text { for } 1 \neq 0
\end{aligned}
$$

It is useful in computations to note from Eqo (4) that:

$$
\sum_{q=1}^{I+1} A_{1 j q}=8_{01}
$$

The quantitifes $\mathrm{N}_{j}(0)$ and $\mathrm{C}_{1 j}(0)$, are determined from the initial conditions... If a neutron is injected at $x=0, t=0$ then $n(x, 0)-\delta(x-0)$, and 
$N_{0}(0)=L^{-1}, N_{j \neq 0}(0)=2 L^{-1}$, while $C_{i j}(0)=0$. If an $i$-th type precursor. is injected at $x=0, t=0$ then $C_{i 0}(0)=L^{-1}, C_{i j}(0)=2 L^{-1}$ for $j \neq 0$, and all other coefficients vanish initially。 Let $n_{i}(0,0 ; x, t)$ represent the neutron density at $x, t$ resulting from injection at $x=0, t=0$ of a neutron $(I=0)$ or an i-th type precursor $(i \neq 0)$ 。 Then combining the initial conditions, $\mathrm{Eq}_{0}(4)$ and Eqs. (2) we find:

$$
\operatorname{Ln}_{1}(0,0 ; x, t)=\sum_{q=1}^{I+1}\left[A_{10 q} e^{a_{0 q} t}+2 \sum_{j=1}^{\infty} A_{i j q} e^{a_{j q} t} \cos j 2 \pi \frac{x}{L}\right] .
$$

The neutron density in the ring reactor resulting from a burst of arbitrary distrribution and composition (neutrons and/or precursors) can be constructed by superposition of the basic solutions Eq. (8).

\section{III。 SPECIAL CASES}

\section{A. Delayed Neutrons Absent}

If it is assumed that delayed neutrons are not formed in fissions, i。e, $k_{1}=k_{2}=\ldots \ldots$ m $k_{I}=0$, then Eq. (B) reduces lo a slmple form. From Eqs。(6), $\mathrm{A}_{\mathrm{Ojq}}=1$, and from Eq. (5) there is only one root $a_{j l}$ in place of the $I+I$ roots in $\mathrm{Eq}$. (8). In this case $\mathrm{Eq}$. (8) becomes:

$$
\operatorname{Ln}_{0}(0,0 ; x, t)=e^{\frac{k_{0}-1}{T^{2}}} t\left[1+2 \sum_{j=1}^{w} e^{-j^{2} 4 \pi^{2} \frac{M^{2}}{L^{2}} \frac{t}{\tau_{a}}} \cos j 2 \pi \frac{x}{L}\right]
$$

The bracketed expression in $\mathrm{Eq} \cdot$ (9) is just the function $\theta_{3}\left(\frac{\mathrm{x}}{\mathrm{L}}, \sqrt{-I} 4 \pi \frac{\mathrm{M}^{2} \mathrm{t}}{\mathrm{L}^{2} \tau}\right)$ where the theta-function is defined by: 


$$
\theta_{3}(\xi, \eta) \equiv I+2 \cdot \sum_{j=1}^{\infty} e^{\sqrt{-1} \pi \eta j^{2}} \cos 2 \pi j \xi
$$

Eq. (9) can be regarded as the product of an amplitude factor

$$
\left(k_{0}-1\right) t \tau_{a}^{-1}
$$

and a shape function

$$
\theta_{3}\left(\frac{x}{L} ; \sqrt{-1} 4 \pi \cdot \frac{M^{2} t^{2}}{L^{2} \tau}\right)
$$

The shape function is graphed in Fig. 1 as a function of position at various times for a.model resembling a large, annular, water-moderated reactor. The diameter of the ring is taken to be $4 \mathrm{ft}$, the migration length is $6.1 \mathrm{~cm}$, and the mean time for neutron loss $\tau_{a}$ is $10^{-4} \mathrm{sec}_{\text {o }}$ Values of the $\theta_{3}$-function were obtained from Jahnke-Ende. 2/... It can be seen from the figure that the neutron density is nearly equalized around the ring at a time about $1 / 25$ sec after the burst。

A general expression can be obtained from the tables for the time $t_{\text {eq }}$ required for equalization. (within 3\%) of the neutron density around the ring. This expression is:

$$
t_{\text {eq }} \cong \frac{I}{1 \sigma}\left(\frac{L}{M}\right)^{2} \tau_{a}
$$

A.t times much shorter than the equalization time the neutron density is distributed much as a Gaussian function. This is physically reasonable when the disturbance has not spread far from $x=0$. The observation follows analytically from the fact. that the $\theta_{3}$-function, and indeed our entire solution, can be built up by superposing.. the infinite medium (Gaussian) distributions resulting from an array of simultaneous bursts situated at $\ldots \ldots,-2 \mathrm{~L},-\mathrm{L}, 0, \mathrm{~L}, 2 \mathrm{~L}, \ldots \ldots$. 


\section{B. Critical Reactor With One Delayed Neutron Group}

When even one delayed neutron group is introduced the situation becomes much more complicated. In order to simplify the computation of Eq. (4) it. is assumed that the reactor is just critical, $i_{0} \theta_{0}, k_{0}+k_{1}=1_{0}$. Moreover. It. Is assumed that the various parameters involved in the a-equation, $\mathrm{Eq}_{0}$ (5), are such thet $\tau_{\theta} \lambda_{1}$ is smail compared with $k_{1}$ and with $\pi^{2} M^{2} / L^{2}$. With these provisions Eqs... (5) and (6) can be solved approximately to yield for all values of $j:$

$$
\begin{aligned}
a_{j 1} \cong-k_{1} \tau_{a}^{-1}-4 \pi^{2}{ }^{2} M^{2} / L^{2} ; a_{j 2} \cong-\lambda_{1} /\left(1+\frac{k_{1} L^{2}}{4 \pi^{2} M^{2} j^{2}}\right) & \\
A_{0 j 1}=1-A_{0 j 2} \quad & ; A_{0 j 2} \cong \frac{T_{8} \lambda_{1}}{k_{1}}\left(1+4 \pi^{2}{ }^{2} M^{2} / k_{1} L^{2}\right)^{-2}
\end{aligned}
$$

When these approximate forms are inserted into $\mathrm{Eq}$. (4) the result is:

$$
\begin{aligned}
& \operatorname{In}_{0}(0,0 ; x, t) \cong e^{\frac{k_{0}-1}{T_{a}} t} \theta_{5}\left(\frac{x}{\tau_{1}}, \sqrt{-1} \cdot \frac{M^{2} t_{t}}{L^{2} \tau_{a}}\right) \\
& +\frac{\tau_{e} \lambda_{1}}{k_{1}}\left\{i-e^{-k_{1} \tau^{-1}+t_{1}}+2 \sum_{j=1}^{\infty} \frac{1}{\left(1+4 \pi_{j}^{2} M^{2} / k_{1} L^{2}\right)^{2}} \cos j 2 \pi \frac{x}{L}\right. \\
& \left.\left(-\frac{\lambda_{1} t}{1+\left(k_{1} L^{2}\right) / 4 \pi^{2} M^{2} j^{2}}-k_{1} \tau^{-1} t-4 \pi^{2} j^{2} \frac{M^{2} t}{L^{8} \tau}\right)\right\}
\end{aligned}
$$

The result has been rearranged to isolate the tabulated $\theta_{3}$-function. 
The term in Eq。(13): which contains the theta-function represents the "prompt decay,". and because $\tau_{8} \lambda_{1} / k_{1} \ll 1$ by assumption, this term dominates the neutron distribution for some time after the burst.. The "prompt decay" term rapidly becomes roughly equalized and then dies away. During the' "prompt decay" a store of delayed neutron precursors has been built up, particularly in the vicinity of the burst, and these precursors subsequently act as a distributed neutron source: Because the precursor distribution is not uniform, the resulting neutron distribution cannot be expected to be uniform, and the equalization time or time required for decay of the non-uniformity can be expected to be of the order of the mean precursor decay time.

These observations are illustrated in Fig. 2 where, as in the previous case $M=6.01 \mathrm{~cm}, \tau_{a}=10^{-4} \mathrm{sec}$ and the diameter of the ring is $4 \mathrm{ft}_{0}$. The single delayed neutron group at our disposal is given characteristics representing an average of all delayed groups emitted from fission, $i_{0} e_{0}, k_{1}=0.007$ and $\lambda_{1}=0.07 \mathrm{sec}^{-1}$. The neutron densities at the point of burst injection and at the opposite point of the ring are graphed against time in Fig。2。 The approximate equalization and rapid decey of the "prompt" contribution are evident, while the subsequent non-uniformity of neutron density is also shown. From the form of the bracketed terms of $\mathrm{Eq} 。(13)$ we can estimate the mean life of the non-uniformity as $\left(1+k_{1} L^{2} / 4 \pi^{2} M^{2}\right) \lambda_{1}^{-1}$ or about $24 \mathrm{sec}$ for the case illustrated in Fig. 2。

\section{DISCUSSION}

An annular reactor in which free neutron motion is restricted by a fully-absorbing, central island has some features similar to those of the simpler ring reactor. In particular, azimuthal propagation of disturbances in a large 
annular reactor has been investigated by studying the similar problem for a ring reactior. The study has been facilitated by the fact that a major part of the solution involved the tabulated $\theta_{3}$-function, an unusual situation in nonseparable problems. The results perhaps can be summarized best in terms of the : equalization time $\left(t_{e q}\right)$ required for decay of a non-uniformity in neutron distribution. Two such times can be distinguished. The major portion of the non-uniformity is removed in the "prompt decay," and the time $t_{\text {eq }}$ (prompt) required for this process has heon obtainod in Eection III-A. Final equalization results only after decay of the precursors produced by the original non-uniformity in neutron distribution. The time required for final equalization (to within 3\%) can be estimated from Eq. (13) for the case when one delayed neutron group is present and the reactor is critical. The two equalization: times are given. in Eq. (14)。

$$
\begin{gathered}
t_{\text {eq }}(\text { prumpl }) \cong \frac{1}{10}\left(\frac{\mathrm{L}}{\mathrm{M}}\right)^{2} \tau_{a} \\
\therefore \\
t_{\text {eq }}\left(\text { deleyeju) } \cong\left(1+\xi^{-1}\right) \lambda_{1}^{-1} \ln \frac{18 \mathrm{~g}}{(1+\xi)^{2}}\right.
\end{gathered}
$$

where $\xi$ is defined as $4 \pi^{2} \mathrm{M}^{2} / \mathrm{L}^{2} \mathrm{k}_{1}$. The two times can be very different; for the case presented in Section III, $t_{\odot q}$ (prompt) is about $1 / 25$ sec while $t_{\text {eq }}$ (delayed) is about 78 sec。

\section{ACKNOWLEDGMENT}

The author is grateful to $R$. $R$. Schiff of Bettis Laboratory for suggesting the problem. 


\section{REFERENCES}

1.. H. Soodak, Ch。 8, "The Science and Engineering of Nuclear Power," Vol. II, Addison Wesley Press, Cambridge, Mass。; (1949)。

2. E. Jahnke and F. Fmde, "Tables of Functions," Dover Publications, New York (1945)。 


\section{SHAPE FUNCTION OF PROMPT NEUTRON} DISTRIBUTION RESULTING FROM NEUTRON BURST

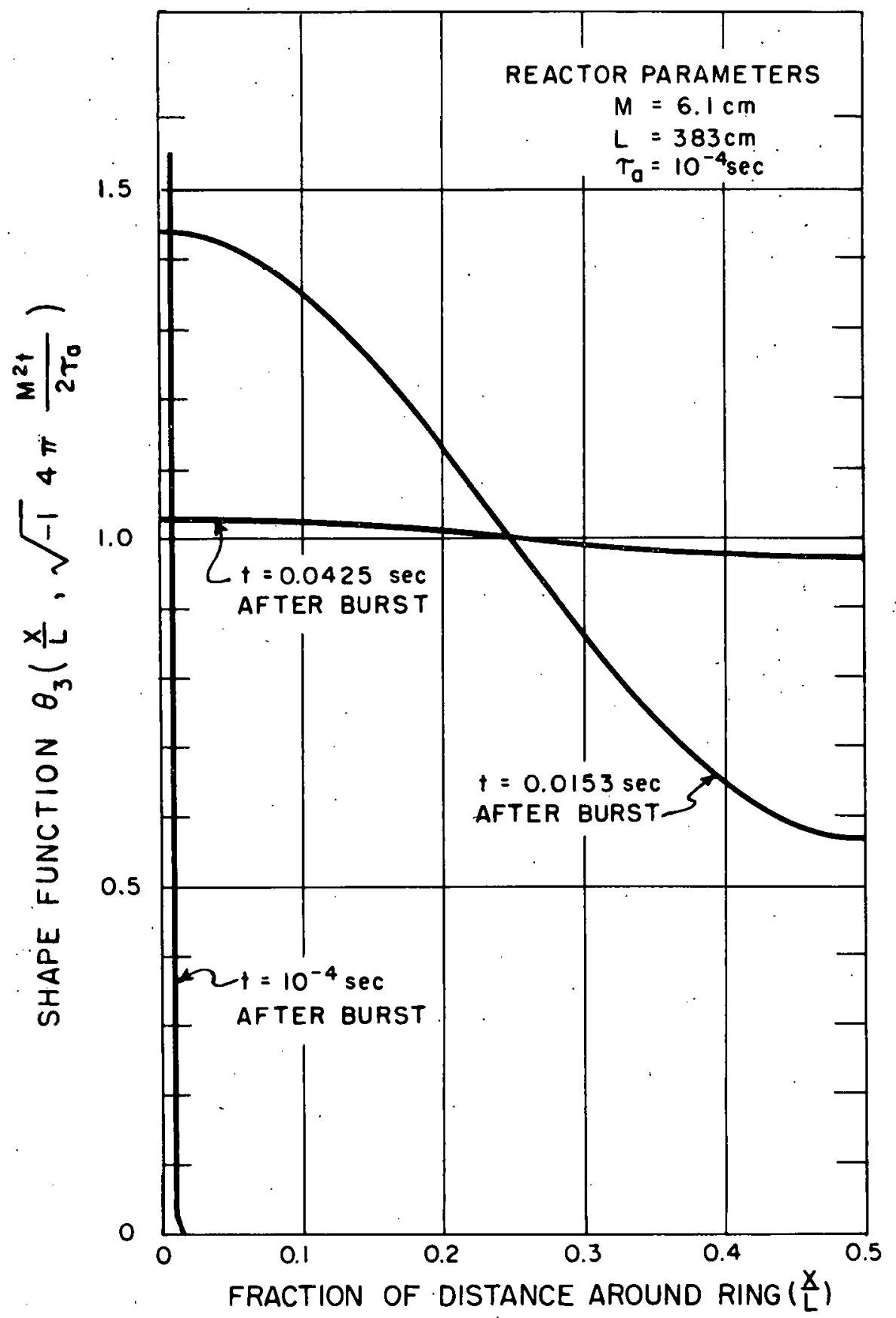

Fígure 1 
NEUTRON DENSITY AT POINT OF NEUTRON BURST AND AT OPPOSITE SIDE OF RING REACTOR VERSUS TIME AFTER BURST

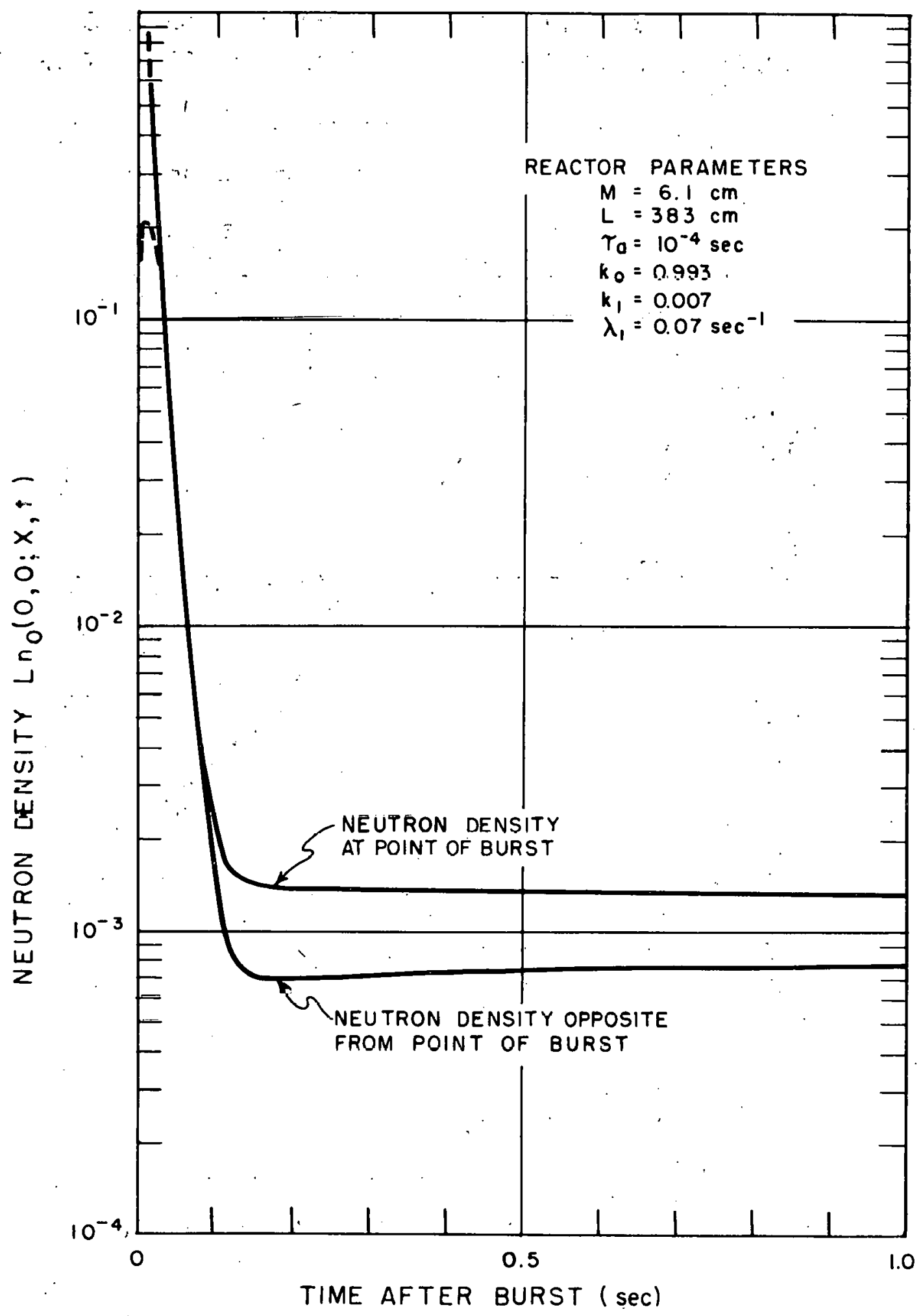

Figure 2 